\title{
Abnormalities in synovial fluid of patients with septic arthritis detected by gas-liquid chromatography $\frac{\frac{6}{6}}{\frac{\bar{m}}{6}}$
}

\author{
ITZHAK BROOK, MICHAEL J. REZA, KENNETH S. BRICKNELL, \\ RODNEY BLUESTONE, AND SYDNEY M. FINEGOLD
}

From the Infectious Disease and Rheumatology Sections, Wadsworth VA Hospital and Department of Medicine, $\vec{\omega}$ UCLA Medical Center, Los Angeles, California, and Children's Hospital National Medical Center, Washington, $D C, U S A$

SUMMARY Gas liquid chromatography was performed on synovial fluid of 94 patients with exudative $\underset{\infty}{\vec{\Omega}}$ arthritis. A relatively constant pattern of peaks was obtained in all synovial fluid samples. Lactic을 acid was increased in synovial fluid of patients with septic arthritis excluding gonococcal arthritis. $\vec{\rightarrow}$ In addition 2 constant peaks with retention time of 546 and 848 seconds in the chromatogram usuallyo showed a significant increase in the synovial fluids of patients with septic arthritis (including gonococcal arthritis) but not in those from patients with sterile inflammatory or degenerative arthritis. $\odot$ In 1 patient with pseudomonas arthritis an increase in the peaks was noted with clinical and bacterio-. logical relapse and subsequently there was a gradual return to normal levels during clinical improve-O ment. Increase in synovial fluid lactic acid is useful in the diagnosis of septic arthritis. Identification of the compounds represented by the 2 peaks, which presumptively correspond to n-valeric and $\bar{D}$ n-hexanoic acid, may provide further information on diagnosis, prognosis and pathogenesis of $\frac{\circ}{\otimes}$ arthritis and may help in the differentiation between gonococcal and nongonococcal arthritis.

The early and accurate diagnosis of septic arthritis is of great clinical importance. Without treatment bacterial infection can cause rapid and complete destruction of joints. Differentiation of an infectious arthritis from a noninfectious inflammatory synovitis is a frequent diagnostic problem for physicians. In particular, patients who present with an acute and dominant monoarticular arthritis, with or without a past history of a chronic polyarthritis, should always be suspected of having a septic arthritis. Yet synovial fluid analysis may fail to yield a diagnosis despite careful bacteriological examination. Moreover, in addition to a sharp increase in the incidence of gonococcal arthritis we have recently seen several patients with coexisting acute gouty and septic arthritis.

Concentrations of synovial fluid lactic acid in various pathological conditions have been measured in the past (Treuhalt and McCarty, 1971). However, almost all the data were related to patients suffering

Accepted for publication 30 April 1979

Correspondence to Itzhak Brook, MD, Clinical Microbiology Laboratories and Infectious Diseases, Children's Hospital National Medical Center, 111 Michigan Avenue, NW, Washington DC 20010, USA. from noninfectious inflammatory processes (Roberts? et al., 1967).

Recent work done by various investigators has $\stackrel{0}{\circ}$ shown that increased amounts of lactic acid and $\frac{5}{3}$ volatile fatty acids in cerebrospinal fluid indicated the presence of bacterial meningitis (Bland et al.,,$\frac{\rho}{3}$ 1974; Controni et al., 1975; Brook et al., 1976; Brook et al., 1978a). This test was proved to be very reliable in differentiating aseptic from bacterial $\$$ meningitis, even in partially treated cases. We were thus stimulated to study the concentrations of $N$ synovial fluid lactic acid and volatile fatty acids in $N$ patients with active exudative arthritis to see if there tests could be of value in the rapid diagnosis of ${ }_{\sigma}^{\omega}$ septic arthritis.

\section{Materials and methods}

Ninety-four patients with a diagnosis of untreated, $\frac{T}{0}$ acute monoarticular arthritis or chronic arthritis with an exacerbation limited to a single joint were $\frac{?}{\mathbb{P}}$ admitted to Wadsworth Hospital Center, Veterans $\supseteq$ Administration, the University of California, Los? Angeles Medical Center, or the Children's Hospitalo National Medical Center, Washington, DC, between? 
1 August 1976 and 1 January 1978. Seventy-seven of the patients were males, and 17 were females. Their ages ranged from 5 to 66 years, average 32 years. On admission and before any new therapy was given the affected joints were aspirated. Repeated aspiration was performed during the course of therapy in several patients, as indicated by the clinical course.

The patients were classified in 3 major diagnostic subgroups: septic arthritis, inflammatory sterile arthritis, and degenerative arthropathy. The specific diagnostic entities are noted in Table 1.

\section{GROUP 1: SEPTIC ARTHRITIS}

In addition to positive synovial fluid bacterial cultures patients in this group tended to have depressed synovial fluid glucose concentrations in the presence of increased leucocytes (especially polymorphonuclear leucocytes). Of the 12 patients with gonococcal arthritis 8 had positive synovial cultures for gonococci, while 4 had the diagnosis established on the basis of positive urethral cultures for gonococci, tenosynovitis, pustular skin rash, and rapid response to intravenous penicillin. The causative organisms are listed in Table 1.

\section{GROUP 2: STERILE INFLAMMATORY}

\section{ARTHRITIS}

Patients with the following diagnoses presented with an acute exacerbation of arthritis, negative bacterial cultures, and clinical, radiographic, and

Table 1 Diagnosis in 94 patients with exudative arthritis, solely or largely confined to a single joint

\begin{tabular}{lr}
\hline Diagnosis & Number of patients \\
\hline 1. Septic arthritis & 12 \\
Neisseria gonorrhoeae & 14 \\
Staphylococcus aureus & 6 \\
Pseudomonas aerugino sa & 9 \\
Other Gram-negative bacilli & \\
(Klebsiella 2; Proteus mirabilis 2; & \\
$\quad$ Enterobacter 1; H. influenzae 3; & \\
$\quad$ and E. coli 1) & 1 \\
Anaerobic streptococcus & 1 \\
Streptococcus pneumoniae & 1 \\
Candida albicans & 44 \\
Total & \\
2. Sterile inflammatory arthritis & 17 \\
Rheumatoid arthritis & 9 \\
Acute gout or pseudogout & 8 \\
Traumatic & 5 \\
Chronic inflammatory bowel disease & 1 \\
Reiter's syndrome & 1 \\
Psoriatic arthritis & 1 \\
Systemic lupus erythematosus & 42 \\
Total & \\
3. Degenerative arthropathy & \\
Osteoarthritis & \\
Total & \\
\hline
\end{tabular}

biochemical evidence of the conditions listed here below: Rheumatoid arthritis (Ropes et al., 1958) (17 patients); acute gout and pseudogout (crystal proved) (9 patients); traumatic arthritis-patients with this diagnosis had acute trauma to their joints within the 48 hours of the time seen and presented with joint effusions, usually bloody ( 8 patients); chronic inflammatory bowel disease (5 patients); Reiter's syndrome (HLA B27-antigen positive) (1 patient); psoriatic arthritis (1 patient); systemic lupus erythematosus (1 patient).

\section{GROUP 3: DEGENERATIVE ARTHROPATHY}

Patients with osteoarthrosis complicated by small, transient, and largely noninflammatory effusions (class 1 fluids) were studied (8 patients).

Synovial fluid was obtained by direct puncture of the affected joints under local anaesthesia by aseptic technique. $0.1 \mathrm{ml}$ of $50 \%$ sulphuric acid was added to $0.9 \mathrm{ml}$ of the synovial fluid immediately after collection. Specimens were placed in a sterile tube and frozen immediately at -20 degrees $C$ until analysed.

Lactic acid measurement was done as previously described by gas-liquid chromatography (GLC) (Bricknell et al., 1979). $1 \mu \mathrm{l}$ of aqueous supernatant of the synovial fluid was injected for detection of volatile fatty acids. An instrument with a flame ionisation detector was used for this analysis. The column was packed with FFAP 6\% (Varian Aerograph, Sunnyvale, CA 94086). The gases used were nitrogen $30 \mathrm{ml} / \mathrm{min}$, hydrogen $20 \mathrm{ml} / \mathrm{min}$, and air $300 \mathrm{ml} / \mathrm{min}$. The temperature of the injection block was $210^{\circ} \mathrm{C}$, that of the column over $200^{\circ} \mathrm{C}$, and of the detector block $215^{\circ} \mathrm{C}$. A standard mixture prepared as described in the VPI Anaerobic Laboratory Manual (Holdeman et al., 1977, p. 135) of lactic acid, volatile fatty acids, and alcohol was used for presumptive identification. The standard mixture was acidified and treated in the same manner as the synovial fluid.

\section{Results}

Lactic acid concentrations were significantly increased in the synovial fluids of patients with septic arthritis excluding gonococcal arthritis. The data concerning the lactic acid findings have been previously reported (Brook et al., 1978b).

Eight other chromatographic peaks with retention times (RT) of 78, 92, 134, 205, 250,363, 546, and 848 seconds were consistently noted. Two of these peaks (546 and 848) appeared to correlate with septic arthritis in a number of patients. In addition peaks with retention times of 163,663 , and 1815 seconds were inconsistently noted. 
170 Brook, Reza, Bricknell, Bluestone, Finegold

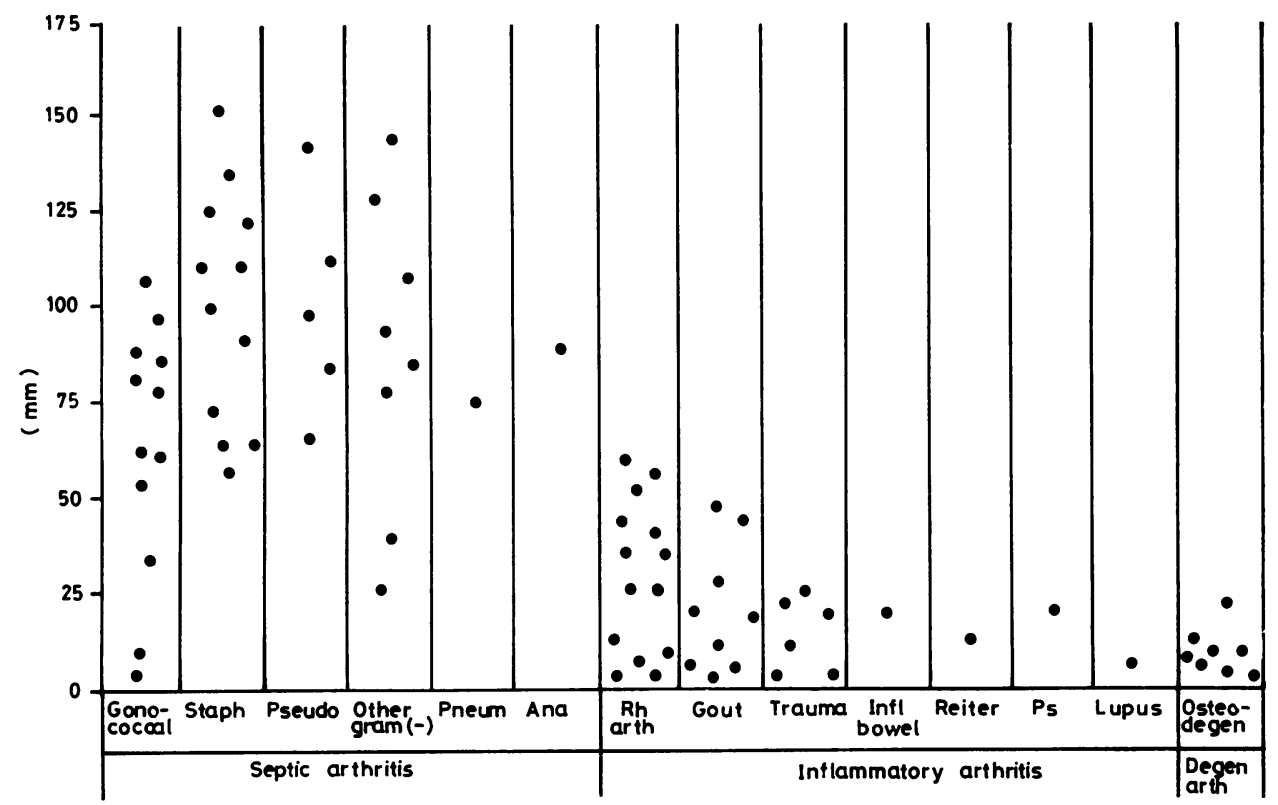

Fig. 1 Comparison of levels of the compound with retention time of 546 seconds in 94 patients with exudative arthritis. Gonococ-Gonococcal. Staph-Staphylococcus aureus. Pseudo-Pseudomonas. Other gram (-)-Other Gram-negative rods. Pneum-Streptococcus pneumoniae. Ana-Anaerobic Gram-positive cocci. Rh arth-rheumatoid arthritis. Inf Bowl_Inflamatory bowel disease. Reiter-Reiter's syndrome. Ps-Psoriatic arthritis. Osteo-degendegenerative arthropathy.

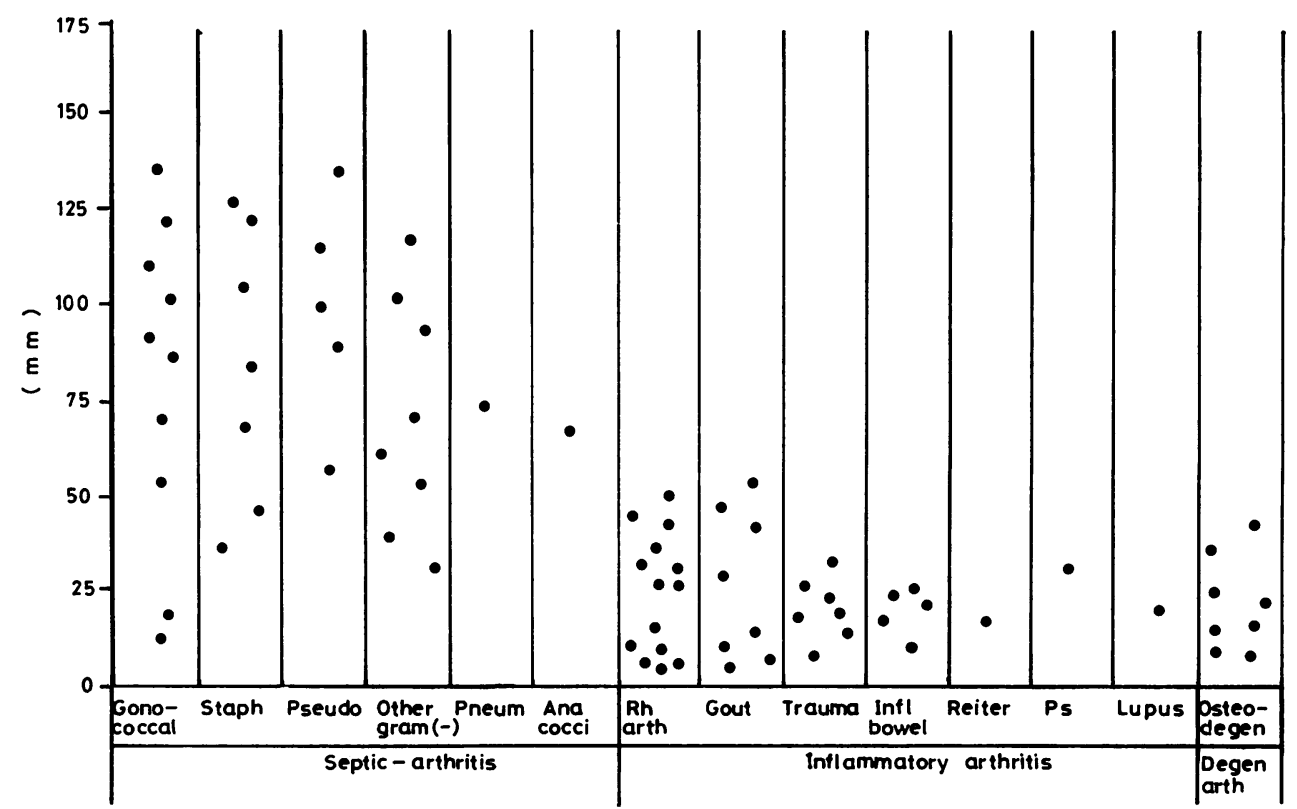

Fig. 2 Comparison of levels of the compound with retention time of 546 in 94 patients with exudative arthritis. For abbreviations see Fig. 1 . 
The distribution of the 546 seconds RT peak according to diagnosis is shown in Figure 1. This compound corresponds in its retention time to $\mathrm{n}$-valeric acid in the standard used. Statistical analysis by Student's $t$ test showed that there was a highly statistically significant difference $(P<0.001)$ beween the mean concentration of this compound in septic (group 1) and both the sterile inflammatory (group 2) and degenerative fluids (group 3) (group 1 , mean 83.91 $\pm 6.2 \mathrm{SD}$, versus group 2 , mean $26 \cdot 7 \pm 1 \cdot 9$, and group $3,26 \cdot 2 \pm 4 \cdot 4$ ). However, there was overlap between patients in the first and the second 2 groups.

The compound with a retention time of 848 seconds shows a similar distribution (Fig. 2). The retention time of this compound corresponds to the retention time of $n$-hexanoic acid in the standard solution used. Student's $t$ test showed that there was a highly statistically significant difference $(P<0.001)$ between the mean concentration of this compound in the septic group (group 1 , mean $85 \cdot 3 \pm 5 \cdot 6$ ) versus the sterile inflammatory (group 2, mean $28 \cdot 4 \pm 3 \cdot 1$ ) and degenerative fluids (group 3 , mean $16 \cdot 6 \pm 1 \cdot 8)$.

The peaks with retention times of 78, 92 and 134, 205,250 , and 363 seconds did not correlate with any specific diagnosis.

The pattern of progression of all the peaks including that of lactic acid during the course of treatment of a patient with septic arthritis caused by Pseudomonas aeruginosa is illustrated in Fig. 3. Maximal concentrations were reached between days 7 and 9 . Values fell slowly to baseline values by the four- teenth day. The patient had a recurrence of the infection on day 17, with concomitant elevation of the volatile fatty acids and lactic acids peak in the synovial fluid.

\section{Discussion}

Increased amounts of lactic acid and 2 compounds with GLC retention times of 546 and 848 seconds within the synovial fluid of patients with septic arthritis have been found. Studies of other GLC peaks revealed unpredictable changes which had no apparent correlation with the presence or absence of infection. The changes in the 2 compounds were similar to those previously reported for lactic acid concentrations (Brook et al., 1978b) except in gonococcal arthritis.

It is of interest that the changes noted in volatile substances in septic arthritis are in the long retention time area, whereas those observed in bacterial meningitis were noted in the short retention time area (Brook et al., 1978a). The sensitivity of these changes was most clearly seen in a single patient with recurrent septic arthritis, in whom increases in these substances occurred concomitantly with clinical relapse. Since the changes in certain volatile substances in the presence of septic arthritis may persist after 3 to 4 days of treatment, they may be helpful in differentiating partially treated septic arthritis from other types of sterile inflammatory arthritis. However, there was much overlap of individual values from patients with septic arthritis and other types of inflammatory arthritis in

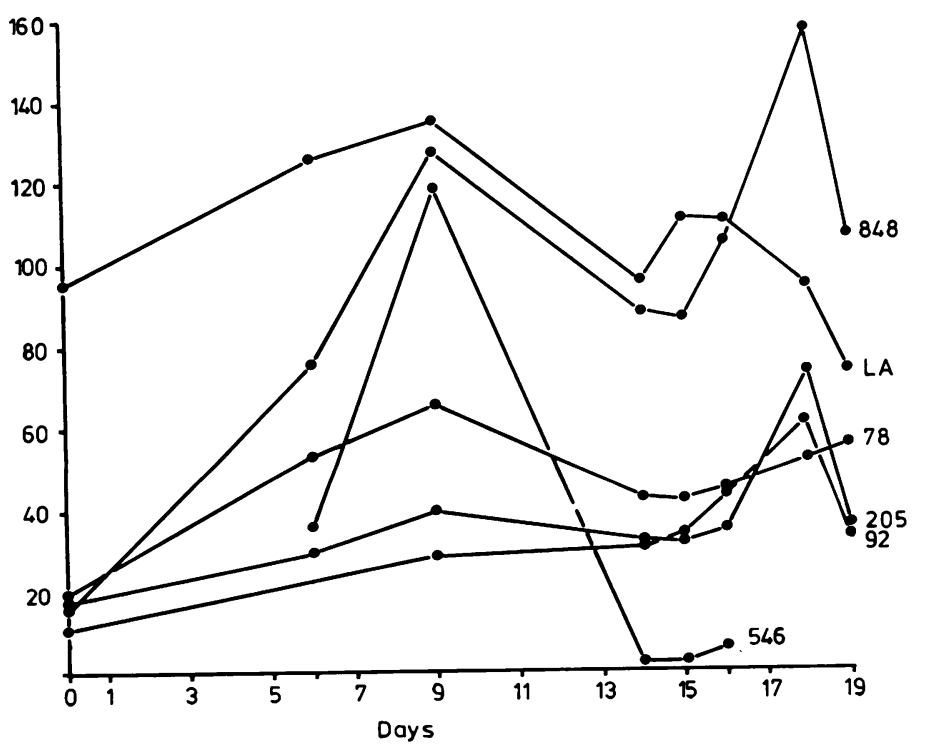

Fig. 3 The serial changes in volatile fatty acids and lactic acid in a patient with pseudomonas arthritis. This 39-year-old female had a serious Pseudomonas aeruginosa septic arthritis of the knee. Synovial fluid cultures were positive through day 9, transiently negative days 10-13, then positive again on days 14-18. Recovery was temporally related to intra-articular therapy with $15 \mathrm{mg}$ of tobramycin $(T) . G$ and $C$-gentamicin and carbenicillin IV.G-intra-articular gentamicin. A-afebrile. 
contrast to lactic acid values, where no such overlap exists.

The data presented suggest that lactic acid and certain volatile substances with relatively long retention times can be used as an additional and valuable diagnostic tool in differentiation between septic and nonseptic arthritis, especially in cases where other diagnostic data are overlapping and are not clear-cut. Lactic acid measurement can clearly differentiate between nongonococcal septic arthritis and other sterile inflammatory and noninflammatory conditions in the joints, and can alert the physician to the presence of a superimposed bacterial infection in patients suffering from any type of chronic polyarthritis.

Measurement of the 2 volatile substances which were almost always increased in cases of septic arthritis (including gonococcal disease), might help in differenting nongonococcal septic arthritis from gonococcal arthritis. In gonococcal arthritis only the levels of the 2 volatile substances were raised, whereas in nongonococcal septic arthritis the levels of both lactic acid and the 2 volatile substances were raised. More work, however, is needed to investigate further the potential clinical usage of gasliquid chromatography in the diagnosis of septic arthritis.

\section{References}

Bland, R. D., Lester, R. D., and Ries, J. P. (1974). Cerebrospinal fluid lactic acid and $\mathrm{pH}$ in meningitis. American Journal of Diseases of Children, 128, 151-156.
Bricknell, K. S., Brook, I., and Finegold, S. M. (1979). Optimizing methylation conditions for gas-liquid chromatography assay of lactic acid in biological samples. Chromatographia, 12, 22-24.

Brook, I., Bricknell, K. S., Overturf, G. D., and Finegold, S. M. (1976). Abnormalities in spinal fluid (CSF) detected by gas-liquid chromatography (GLC) in meningitis patients. Abstracts of Annual Meeting American Society of Microbiology, p. 107.

Brook, I., Bricknell, K. S., Overturf, G. D., and Finegold, S. M. (1978a). Measurement of lactic acid in cerebrospinal fluid of patients with infections of the central nervous system. Journal of Infectious Diseases, 137, 384-390.

Brook, I., Reza, M. J., Bricknell, K. S., Bluestone, R., Finegold, and S. M. (1978b). Joint fluid lactic acid, diagnostic acid in septic arthritis. Arthritis and Rheumatism, 21, 774-779.

Controni, G., Rodriquez, W. J., Deane, C., Ross, S., Khan, W., and Puig, J. R. (1975). Rapid diagnosis of meningitis by gas liquid chromatographic analysis of cerebrospinal fluid lactic acid. Clinical Proceedings of the Children's Hospital National Medical Center, 31, 194-201.

Holdeman, L. V., Elizabeth, P. C., and Mose, W. E. C. (1977). Anaerobic Laboratory Manual, VPI and State University, 4th edn. Blacksburg, Virginia.

Roberts, J. E., McLess, B. D., and Werley, G. P. (1967). Pathways of glucose metabolism in rheumatic and nonrheumatoid synovial membrane. Journal of Laboratory and Clinical Medicine, 70, 503-511.

Ropes, M. W., Bennett, G. A., and Cobb, S. (1958). Revision of diagnostic criteria for rheumatoid arthritis. Bulletin on Rheumatic Diseases, 9, 175-176.

Treuhalt, P. S., and McCarty, D. J. (1971). Synovial fluid $\mathrm{pH}$, lactate, oxygen and carbon dioxide partial pressure in various joint diseases. Arthritis Rheumatism, 14, 475-484. 